\title{
Ley chilena de tolerancia cero al alcohol: fortalezas, falencias y carencias que no deben ser obviadas
}

\author{
LEONARDO GONZÁLEZ-WILHELM ${ }^{1,2, a}$, JENNIPHER JOHNSON ${ }^{1, \mathrm{~b}}$, \\ RAÚL CARNEVALI ${ }^{2, c}$, GUIDO RUIZ ${ }^{3, \mathrm{~d}}$
}

\section{Alcohol zero tolerance law in Chile: strengths, flaws and shortcomings that should not be overlooked}

Chile has recently entered into force Act No. 20.580, which modifies the legal limits of blood alcohol concentration in drivers and increases the penalties for driving under the influence of alcohol, narcotics or psychotropic substances. The aim of this review was to give an account of the strengths of this new law but, at the same time, to alert the scientific and legal community about its flaws. We also present some shortcomings of Chilean regulatory framework that remain uncorrected, those that should be considered in the design of public policies for improving road safety and the criteria that judges should ponder during judgment, to determine either conviction or acquittal.

(Rev Med Chile 2012; 140: 939-945).

Key words: Accidents; Alcohol drinking; Chile; Criminal Law; Legislation.

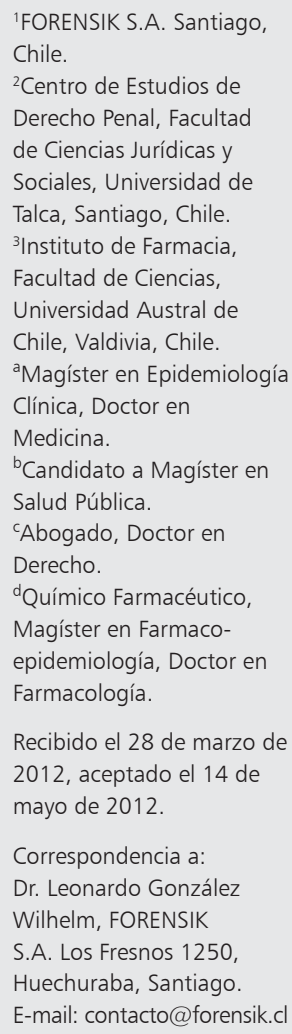

S abido es que las sustancias psicoactivas (sean drogas o fármacos) deterioran la capacidad de conducción de vehículos. En lo relativo al alcohol (en rigor, alcohol etílico o etanol), estudios de casos/controles ${ }^{1}$ han demostrado que su consumo es una causa específica de accidentes de tránsito (AT). Estimaciones indican que a nivel mundial $20 \%$ de todos los AT son debidos al alco$\mathrm{hol}^{2}$, en tanto que la chance de verse involucrado en un accidente aumenta vertiginosamente a partir de concentraciones sanguíneas de etanol (CSE) de $0,4 \mathrm{~g} \% \mathrm{o}^{3-7}$. Es así como con CSE de $0,5 \mathrm{~g} \%$ o el riesgo de un AT mortal se duplica, desde $0,8 \mathrm{~g} \%$ es diez veces más alto; 20 veces mayor a partir de $1,0 \mathrm{~g} \%$ o y 100 veces más a partir de $1,5 \mathrm{~g} \% \mathrm{o}^{4}$.

Estadísticas tanto nacionales ${ }^{8}$ como internacionales ${ }^{9}$ indican que los AT asociados al alcohol ocurren fundamentalmente de noche y durante los fines de semana, siendo causados principalmente por varones, la mayoría de ellos jóvenes. Varios estudios ${ }^{1,10-12}$ dan cuenta además que dichos conductores exhiben una serie de conductas viales que les son típicas (Tabla 1), en virtud de las cuales lo más frecuente es que generen AT producto de conducción a exceso de velocidad o por no mantener correctamente la pista de circulación?

En muchos países las tasas de AT asociados al alcohol han sido reducidas disminuyendo el límite legal aceptable 2 . Es así como desde inicios de la década de 1990-99 países como Suecia, Noruega, España, Suiza o Japón han reducido la CSE que determina una sanción judicial a valores inferiores a $0,5 \mathrm{~g} \%{ }^{13-14}$. Recién este año Chile ha adherido a esa tendencia a partir de la reciente entrada en vigencia de la Ley $\mathrm{N}^{\circ} 20.580$ que aumenta las sanciones por manejo en estado de ebriedad, bajo la influencia de sustancias estupefacientes o psicotrópicas y bajo la influencia del alcohol. Dicho cuerpo legal disminuye de $0,5 \mathrm{~g} \%$ a 0,3 $\mathrm{g} \%$ la concentración con la cual una persona será considerada "bajo la influencia del alcohol" y de $1,0 \mathrm{~g} \%$ a a $0,8 \mathrm{~g} \%$ o la concentración a partir de la cual se le considerará "en estado de ebriedad"15.

El objetivo de la presente revisión es dar cuenta 
Tabla 1. Conductas en la conducción de vehículos motorizados que se asocian al consumo de alcohol

\begin{tabular}{|ll|}
\hline Marcado exceso de velocidad & Velocidad llamativamente baja si alcoholemia es muy alta \\
\hline $\begin{array}{l}\text { Conducción serpenteada } \\
\text { Chocar por detrás a vehículos detenidos o que frenan }\end{array}$ & Seguir de largo en las curvas \\
\hline $\begin{array}{l}\text { Olvidar apagar las luces al estacionar } \\
\text { Chocar o colisionar lateralmente vehículos }\end{array}$ & Provocar topones al realizar maniobras de retroceso \\
$\begin{array}{l}\text { No modificar altura de las luces acorde a estado del tránsito } \\
\text { No señalizar el viraje o hacerlo en dirección opuesta a lo }\end{array}$ & Manejar en la oscuridad sin luces \\
señalizado & Subirse a la cuneta al realizar virajes \\
$\begin{array}{l}\text { Conducir en contra del tránsito o sobrepasando el eje } \\
\text { Utilización irracional y exagerada de la bocina }\end{array}$ & Pasar por alto la luz roja de los semáforos \\
\hline
\end{tabular}

de las fortalezas de esa nueva ley pero a la vez, alertar a la comunidad científica y jurídica sobre ciertas falencias asociadas a ésta. Interesa también hacer presente algunas carencias del marco regulatorio chileno que permanecen sin corrección y que, a nuestro juicio, deben ser consideradas tanto en el diseño de políticas públicas tendientes a incrementar la seguridad vial como en la ponderación judicial de las pruebas que determinan una sentencia, sea condenatoria o absolutoria.

\section{Fortalezas de la nueva ley}

El slogan propuesto por la Comisión Nacional de Seguridad de Tránsito (CONASET) y el Servicio Nacional para la Prevención y Rehabilitación del Consumo de Drogas y Alcohol (SENDA) ha sido "El que maneja no toma". Visto desde esa perspectiva, la nueva ley "de tolerancia cero" ha de ser considerada como un significativo avance, por cuanto en otros países esta estrategia ha demostrado ser exitosa, arraigando en la sociedad el concepto de que la conducción y el consumo de alcohol no deben ir asociados ${ }^{13}$, observándose además una disminución de la ingesta en subgrupos de conductores afectos a la ley ${ }^{16}$.

No obstante lo anterior, debe tenerse presente que el éxito de la ley no está exclusivamente supeditado a un cambio cultural. En efecto: la experiencia internacional indica que no existe una correlación entre el límite legal y la CSE de los conductores fiscalizados. A modo de ejemplo, en Polonia se reportan CSE promedio de entre 1,78 $\mathrm{g} \%$ y $1,87 \mathrm{~g} \% 0^{4,12}$ aun cuando el límite legal es 0,2 $\mathrm{g} \%$. Esto se explica porque la efectividad de este tipo de leyes depende de la percepción que se tenga del riesgo de ser controlado y de la severidad de las penas. $\mathrm{Al}$ respecto, Heifer et al. advierten que la sola ley no permite disminuir la tasa de $\mathrm{AT}^{12}$. Dicha situación ha quedado de manifiesto en estudios daneses y noruegos ${ }^{13}$, como también en análisis económicos publicados en Estados Unidos de Norteamérica ${ }^{17}$. Con todo, se trata de resultados controversiales, en la medida que otras investigaciones han reportado o sugerido una reducción de los AT con resultado fatal asociados al alcohol ${ }^{18-21}$.

Hechas estas consideraciones, resulta posible e incluso prudente entender que el beneficio cultural que puede surgir a partir de la nueva ley poseerá (como lo señalan Chang et al. ${ }^{18}$ ) un efecto limitado, dado lo cual la disminución de la accidentabilidad a largo plazo exigirá programas sostenidos de controles policiales aleatorios ${ }^{2,22-24}$.

\section{Falencias de la nueva ley}

\section{Homologación de "alcotest" con alcoholemia}

La sangre es el material o matriz biológica de elección para demostrar la presencia de sustancias tóxicas en los organismos ${ }^{5,25}$ y los resultados de análisis sobre sangre son los únicos que permiten inferir compromiso agudo por efecto de una droga o fármaco. Este principio toxicológico, si bien elemental, actualmente no se encuentra debidamente resguardado en nuestro marco legal.

Es preciso entender que la interpretación de la concentración de alcohol en aire espirado medida con alcoholímetros digitales ("alcotest") es sumamente distinta de la CSE (alcoholemia). El "alco- 
test" fue diseñado como una prueba de tamizaje cuya finalidad práctica es decidir quiénes deben ser sometidos al examen definitivo: la extracción de sangre y determinación de la alcoholemia. Sin embargo, dicha medición no resulta judicialmente útil para inferir la cantidad de alcohol absorbida en la sangre en un momento determinado, estimándose que sus resultados pueden oscilar entre 18\% y $31 \%$ con respecto al valor real de $\mathrm{CSE}^{26}$.

Si bien en 1976 la primera legislación que reglamentó el uso del "alcotest" ${ }^{27}$ se ajustó correctamente al proceder técnico antes referido, la actual Ley de Tránsito ${ }^{28}$ confunde en su Artículo 111 la real utilidad de dicha prueba, dado que entiende el estado de ebriedad (no así la influencia del alcohol) como una determinada concentración de alcohol "en la sangre o en el organismo", la cual constará "en el informe de alcoholemia o en el resultado de la prueba respiratoria".

Más que enmendar este error, la nueva ley "de tolerancia cero" lo potencia al introducir explícitamente la posibilidad de reemplazar el examen de sangre por una "Prueba respiratoria evidencial", la cual entregaría el resultado en un par de minutos y sin la necesidad de trasladar a la persona fiscalizada a un centro de salud. Tanto es así que el Gobierno ha difundido temerariamente un concepto científicamente equivocado, cual es, que la "alcoholemia y la prueba respiratoria evidencial tendrán igual valor probatorio" 29 .

Cabe aclarar que el "alcotest" puede ser considerado sólo como una orientación de la verdadera CSE y no como un parámetro de certeza, por cuanto:

- La equivalencia teórica planteada originalmente por Harger et al. en 1950 (cantidad de etanol presente en $2.100 \mathrm{ml}$ de aire alveolar = a la contenida en $1 \mathrm{ml}$ de sangre) ha sido discutida y relativizada por muchos autores. A modo de ejemplo Jones et al. plantean que la igualdad recién se produce con $2.300 \mathrm{ml}$ de aire espi$\mathrm{rado}^{30}$, en tanto que Penning et al. asignan un rango de equivalencia que va desde los 700 a los $3000 \mathrm{ml}^{11}$.

- Los resultados del "alcotest" pueden ser influenciados por múltiples factores que no inciden en la alcoholemia. Considérese aquí variables tan aleatorias como la presión atmosférica, temperatura ambiental, temperatura corporal o concentración de glóbulos rojos de la persona.
- Durante la fase de absorción del alcohol el valor obtenido por el "alcotest" es sistemáticamente mayor que la alcoholemia. En efecto: si se realiza un examen de "alcotest" inmediatamente después de ingerido un licor, el resultado suele ser muy elevado no obstante no haya ingresado aún alcohol a la sangre (y por tanto, la alcoholemia del individuo sea $0,00 \mathrm{~g} \%$ ).

- Durante las fases de distribución y eliminación, el valor obtenido por el "alcotest" es sistemáticamente menor que la alcoholemia.

Dado lo anterior y según se muestra en la Tabla 2, aquellos países que han aceptado asignar valores de "alcotest" como prueba judicial del nivel de consumo de alcohol han establecido en su legislación que el valor para sancionar será distinto si proviene de una medición en aire espirado o en sangre $^{14,21}$. Ello no fue considerado así en la nueva ley chilena, situación que creemos, legalmente no resulta susceptible de ser enmendada en el Reglamento de la Ley que aún no ha sido promulgado.

\section{Procedimiento ante "alcotest" negativo}

Un "alcotest" negativo no debería ser utilizado como único parámetro para liberar a una persona de investigaciones posteriores ${ }^{31}$, especialmente en aquellos conductores que presentan signos clínicos de deterioro psicofísico. Esos casos obligan a sospechar la presencia en el individuo de drogas o medicamentos psicoactivos ${ }^{32}$, ya que se sabe que hasta 35\% de discrepancias entre la impresión clínica del estado del conductor y el posterior resultado de la alcoholemia se explican por la presencia de dichas sustancias ${ }^{33}$.

Nuestra ley de tránsito y las modificaciones introducidas por la ley "de tolerancia cero" no se

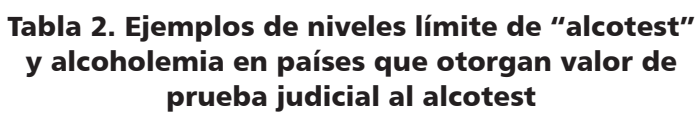
prueba judicial al alcotest

\begin{tabular}{|lcc|}
\hline País & $\begin{array}{c}\text { "Alcotest" } \\
\text { (mg/l) }\end{array}$ & $\begin{array}{c}\text { Alcoholemia } \\
\text { (g\%o) }\end{array}$ \\
\hline Alemania & 0,25 & 0,5 \\
\hline España & 0,25 & 0,5 \\
\hline Holanda & 0,22 & 0,5 \\
\hline Reino Unido & 0,35 & 0,4 \\
\hline Japón & 0,15 & 0,3 \\
\hline
\end{tabular}


hacen cargo de lo anterior y dejan notables vacíos al no indicar en detalle cuándo se deberá sospechar consumo de estupefacientes o psicotrópicos, qué tipo de muestras orgánicas deben ser obtenidas en esos casos (¿sólo sangre? ¿orina? ¿saliva?) ni tampoco la metodología analítica a emplear. Vale tener presente aquí que:

- Los reglamentos de estupefacientes y psicotrópicos $^{34-36}$ incluyen listados muy amplios y heterogéneos, no obstante lo cual, no son excluyentes en la medida que nuestro marco legal prohíbe la conducción bajo los efectos de cualquier sustancia que pueda generar alteraciones en el nivel de conciencia, en la percepción, en la habilidad motriz, en la estabilidad emocional y en el juicio ${ }^{37}$.

- A la fecha ningún test rápido ha demostrado tener niveles de sensibilidad y especificidad aceptables $(>80 \%)$ para la pesquisa de drogas en los controles viales que realiza la policía ${ }^{38}$.

Así por tanto, nuestro marco legal y técnico no otorga lineamientos prácticos concretos para la detección del consumo de estupefacientes y psicotrópicos en conductores. Se abarca mucho sin claridad del cómo proceder, situación que pudiera explicar por qué actualmente las sanciones por consumo de sustancias distintas del alcohol son más bien excepcionales. Asimismo, existe una ventana abierta para condenas injustas en aquellos casos en que los análisis cualitativos arrojen un falso positivo o cuando la sustancia hallada no genera o no indica certeramente un efecto deletéreo sobre la capacidad de conducción (como ocurre por ejemplo con la codeína -un opiáceo- o con el THC-COOH, un marcador inactivo del consumo antiguo de marihuana).

\section{Carencias del marco regulatorio chileno}

\section{Validez científica y certeza jurídica que aporta la alcoholemia}

Cuando se llega a efectuar una alcoholemia, el organismo encargado del análisis es el Servicio Médico Legal (SML). Acorde a la normativa técnica de dicha institución ${ }^{39}$, la medición de la CSE se efectúa mediante cromatografía en fase gaseosa con HeadSpace (CG-HS), lo cual es óptimo dado que dicho examen es el gold standard para la determinación cuantitativa de la alcoholemia ${ }^{40,41}$. Sin embargo, en aquellos SML que no cuentan con cromatografía, el análisis se realiza mediante el micrométodo de Widmark (MW) ${ }^{39}$.

Esto último es problemático ya que el MW hace tiempo se ha abandonado en el campo de la toxicología forense dado que no es específico para etanol (sólo mide sustancias volátiles reductoras). En este escenario, el uso de test alternativos al estándar de referencia es útil sólo si se sabe el riesgo de clasificar mal y éste es aceptablemente bajo $^{42}$. Dicho riesgo se evalúa a partir del conocimiento de las propiedades diagnósticas del test (sensibilidad, especificidad, valores predictivos), las cuales se determinan mediante un proceso de validación de criterio ${ }^{42-45}$. Para el caso del MW un estudio de ese tipo no se encuentra reportado en la literatura, en tanto que el SML sólo utiliza una validación de laboratorio mediante curvas de calibración realizadas a partir de estándares del laboratorio internacional Sigma ${ }^{\circledR}$, alternativa que no otorga suficiente evidencia para apoyar su recomendación ${ }^{46}$ ya que informa sobre la eficacia pero no respecto de la efectividad de la prueba ${ }^{47}$.

Visto lo anterior, ante una alcoholemia positiva mediante el MW se debería corroborar siempre el resultado analizando la muestra con CG-HS. Sin embargo, el SML no efectúa de rutina lo anterior y sólo lo reserva para casos en que se sospeche la presencia de metanol u otros solventes orgánicos ${ }^{39}$.

Cabe hacer presente además que la metodología analítica empleada habitualmente no llega a ser conocida por los intervinientes de un proceso judicial. Contrario a la normativa técnica, lo común es que no se consigne en el informe de alcoholemia si el análisis fue hecho con MW o CG-HS. Asimismo, los peritos de laboratorio del SML no concurren regularmente a declarar a juicios orales en virtud una modificación introducida en 2005 al Código Procesal Penal ${ }^{48}$.

\section{Inexistencia de procedimientos para casos especiales}

Algunas veces los imputados aducen que al momento del AT no habían consumido alcohol y que su ingesta habría tenido lugar con posterioridad al hecho "para calmar los nervios". Esta situación puede ser validada o rechazada pericialmente a partir del análisis de los niveles sanguíneos de metanol ${ }^{10}$-cuestión que rara vez se hace- o bien, procesando simultáneamente muestras de sangre y orina o 2 muestras de sangre obtenidas con un intervalo de 20-30 min, lo cual 
permitirá establecer si el individuo se encontraba en la etapa ascendente o descendente de la curva de eliminación del etanol. Nuestro marco legal, sin embargo, no contempla explícitamente la toma de muestras de orina ni tampoco indica la existencia de situaciones en las cuales sea necesario obtener 2 muestras sanguíneas.

Otra situación más frecuente es que exista retraso entre el AT y el momento de la toma de muestra de sangre para alcoholemia. En dichos casos la obtención de una CSE = 0,00 g\%o no asegura que el individuo no haya estado al momento del hecho bajo los efectos del alcohol. Técnicamente es posible realizar entonces una búsqueda de marcadores de consumo tales como ácidos grasos (ésteres de etanol) o etilglucuronato a nivel de suero, orina o pelo $^{10,11,49}$, procedimientos que pese a su utilidad, no están recogidos en nuestras normativas legales ni técnicas.

\section{Enfermedades y tránsito}

Un tema que se encuentra deficientemente regulado es la incidencia de enfermedades y sus tratamientos en la seguridad vial. Hoy en día el reglamento para la obtención de licencias de conducir ${ }^{37}$ contempla múltiples prohibiciones, algunas genéricas e inespecíficas, otras muy amplias y no acordes al estado actual del conocimiento científico. Asimismo no existen disposiciones legales que regulen situaciones de enfermedad intercurrente. Por ejemplo, en una persona que ha sufrido un infarto cardiaco y evolucionado satisfactoriamente, no existen pautas oficiales que indiquen durante cuánto tiempo un juez deberá considerarlo en condiciones deficientes para la conducción de vehículos motorizados, carencia que adquiere especial relevancia si el paciente protagoniza un AT durante su convalecencia.

\section{Falta de prevención secundaria mediante rehabilitación de conductores alcohólicos}

Quizás uno de los aspectos más deficitarios de la nueva ley es que su enfoque es meramente punitivo. Sabido es que la mayor parte de los conductores involucrados en AT graves asociados al consumo de alcohol poseen CSE que superan con creces los nuevos límites legales establecidos ${ }^{13,14}$. Muchos de ellos son alcohólicos no diagnosticados o no tratados, quienes difícilmente podrán adquirir la conciencia vial que se pretende con la modificación legal.
Sin que el Estado se haya hecho cargo de su rehabilitación, ¿procede aplicarles sanciones (incluso de por vida) a conductores alcohólicos que no han recibido tratamiento?

Lo concreto es que leyes "de tolerancia cero" idénticas a la chilena no han logrado disminuir la tasa de conductores que manejan con CSE $>0,5 \mathrm{~g} \% \mathrm{o}^{13}$. Y aun cuando se castigue con el retiro de la licencia, ello no garantiza una prevención secundaria porque persiste la posibilidad que un alcohólico no rehabilitado siga conduciendo, pero sin licencia. Tal vez sea mejor adoptar un enfoque como el de Alemania, en donde todo conductor que reincide en una falta o bien, es sorprendido con un "alcotest" $\geq 0,8 \mathrm{mg} / \mathrm{l}$ o una alcoholemia $\geq 1,6 \mathrm{~g} \%$, debe completar un programa de rehabilitación impartido por el Estado a fin que le sea devuelta su licencia de conducir ${ }^{14}$.

\section{Conclusiones}

La nueva ley "de tolerancia cero" constituye un avance para arraigar culturalmente el concepto de que conducción y consumo de alcohol no deben ir asociados. Sin embargo, es muy posible que la accidentabilidad no se vea reducida mientras no concurran programas sostenidos de controles policiales aleatorios.

Creemos además que la pretendida "prueba respiratoria evidencial" constituye un error que debe ser revisado previo a la promulgación del reglamento respectivo. Asimismo, advertimos a las autoridades que ciertos temas como validez de la alcoholemia, rol de las enfermedades en materia vial y prevención secundaria en pacientes alcohólicos, no se encuentran aún debidamente abordados en nuestras regulaciones legales y técnicas.

\section{Referencias}

1. Bates MN, Blakely TA. Role of cannabis in motor vehicle crashes. Epidemiol Rev 1999; 21(2): 222-32.

2. Room R, Babor T, Rehm J. Alcohol and public health. Lancet 2005; 365 (9458): 519-30.

3. Borkenstein RF, Crowther RF, Shumate RP, Ziel WB, Zylman R. Die Rolle des alcoholisierten Fahrers bei Verkehrsunfällen ("The Grand Rapids Study"). Blutalkohol. 1974; 11 (Supplement 1): 1-7.

4. Dubey Y, Gujer H-R. Trunkenheit am Steuer im Kanton 
Waadt (Schweiz). Eine Vergleichsstudie über straf- und verwaltungsrechtliche Maßnahmen der Jahre 1970 und 1989 zwischen der Kantonshauptstadt (Lausanne) und einer ländlichen Gegend. Blutalkohol 1993; 30 (5): 26689.

5. Heifer U. Blutalkoholkonzentration und -wirkung, verkehrsmedizinische Charakterisierung und verkehrschtliche Relevanz von Alkoholgrenzwerten im Strassenverkehr. Blutalkohol 1991; 28 (3): 121-45.

6. Odero W, Zwi A. Alcohol-Related Traffic Injuries and Fatalities in Developing Countries: A Critical Review of Literature. Disponible en: http://www.druglibrary.org/ schaffer/Misc/driving/s26p1.htm [Consultado el 27 de marzo de 2012].

7. Movig KL, Mathijssen MP, Nagel PH, van Egmond T, de Gier JJ, Leufkens HG, et al. Psychoactive substance use and the risk of motor vehicle accidents. Accid Anal Prev. 2004; 36 (4): 631-6.

8. Comisión Nacional de Seguridad de Tránsito (CONASET). Siniestros causados por la presencia del Alcohol (2010). Disponible en: http://www.conaset.cl/userfiles/ files/alcohol_total_2010.pdf [Consultado el 27 de marzo de 2012].

9. Haffner HT, Erath D, Kardatzki B. Alkoholtypische Verkehrsunfälle als zusätzliche Beweisanzeichen für relative Fahruntüchtigkeit. NVZ 1995; 8: 301-3.

10. Gilg T. Alkoholbedingte Fahruntüchtigkeit. Beurteilung und Begutachtung in der forensischen Praxis, Teil 2. Rechtsmedizin 2005; 15 (2): 97-112.

11. Alkohol und Verkehrssicherheit. En Penning R., Editor. Rechtsmedizin systematisch. Bremen, Alemania: Editorial UNI-MED; 2006. p. 217-39.

12. Heifer U, Neuhausen M, Pluisch F, Schyma C. Alkohol und Straßenverkehrssicherheit-Untersuchungen zur Epidemiologie und verkehrsrechtlichen Angleichung in Europa. Blutalkohol $199 ; 29$ (1): 1-52.

13. Assum T. Reduction of the blood alcohol concentration limit in Norway-effects on knowledge, behavior and accidents. Accid Anal Prev 2010; 42 (6): 1523-30.

14. González-Wilhelm L, Orellana JJ, Bratzke H. Prävalenz von Alkohol in Blutproben von Erwachsenen bei Verkehrsverstößen in Frankfurt am Main und Südhessen. Querschnittstudie (1993-2004). Blutalkohol 2007; 44 (4): 215-30.

15. Ley 20.580. Publicada en Diario Oficial el 15 de marzo de 2012. Disponible en: http://www.leychile.cl/Navegar ?idNorma $=1037847 \&$ buscar $=20.580$ [Consultado el 28 de marzo de 2012].

16. Chamberlain E, Solomon R. Zero blood alcohol concentration limits for drivers under 21: lessons from Canada. Inj Prev 2008; 14 (2): 123-8.
17. Freeman DG. Drunk driving legislation and traffic fatalities: new evidence on BAC 08 laws. Contemporary Economic Policy 2007; 25 (3): 293-308.

18. Chang $\mathrm{K}, \mathrm{Wu} \mathrm{CC}$, Ying YH. The effectiveness of alcohol control policies on alcohol-related traffic fatalities in the United States. Accid Anal Prev 2012; 45 (2): 406-15.

19. Hingson R, Heeren T, Winter M. Lowering state legal blood alcohol limits to $0.08 \%$ : the effect on fatal motor vehicle crashes. Am J Public Health 1996; 86 (9): 1297-9.

20. Shults RA, Elder RW, Sleet DA, Nichols JL, Alao MO, Carande-Kulis VG, et al. Reviews of evidence regarding interventions to reduce alcohol-impaired driving. Am J Prev Med 2001; 21 (4 Suppl): 66-88.

21. Imai $H$. The new traffic law and reduction of alcohol related fatal crashes in Japan. Inj Prev 2003; 9 (4): 382.

22. Mann RE, Macdonald S, Stoduto LG, Bondy S, Jonah $B$, Shaikh A. The effects of introducing or lowering legal per se blood alcohol limits for driving: an international review. Accid Anal Prev 2001; 33 (5): 569-83.

23. The National Center on Addiction and Substance Abuse (CASA). Teen tipplers: America's underage drinking epidemic. Disponible en: http://www.casacolumbia.org/ articlefiles/379-Teen\%20Tipplers.pdf [Consultado el 27 de marzo de 2012].

24. Rehn N, Room R, Edwards G. Alcohol in the European Region: Consumption, harm, and policies. Copenhagen, Denmark: WHO Regional Office for Europe; 2001.

25. Augsburger M, Donze N, Menetrey A, Brossard C, Sporkert F, Giroud C, et al. Concentration of drugs in blood of suspected impaired drivers. Forensic Sci Int 2005; 153 (1): 11-5.

26. Repetto M. Toxicología avanzada. Madrid, España: Ediciones Díaz Santos; 1995. p. 425-75.

27. Decreto 430, Ministerio de Justicia. Publicado en Diario Oficial el 27 de febrero de 1976. Disponible en: http:// www.leychile.cl/Navegar?idNorma $=125890 \&$ buscar $=\mathrm{de}$ creto+430+1976 [Consultado el 28 de marzo de 2012].

28. Decreto con fuerza de ley 1 , Ministerio de Transportes y Telecomunicaciones; Ministerio de Justicia. Publicado en Diario Oficial el 29 de octubre de 2009. Disponible en: http://www.leychile.cl/Navegar?idNorma $=1007469 \&$ idVersion=2009-10-29 [Consultado el 28 de marzo de 2012].

29. Nueva ley de Tolerancia 0 al alcohol en la conducción. Disponible en: http://www.gob.cl/especiales/nueva-leyde-tolerancia-0-al-alcohol-en-la-conduccion/ [Consultado el 22 de marzo de 2012].

30. Jones AW, Jonsson KA, Kechagias S. Effect of high-fat, high-protein, and high-carbohydrate meals on the pharmacokinetics of a small dose of ethanol. Br J Clin Pharmacol 1997; 44 (6): 521-6. 
31. Mørland J, Beylich KM, Bjørneboe A, Christophersen AS. Driving Under the Influence of Drugs: An Increasing Problem. Disponible en: http://casr.adelaide.edu. au/t95/paper/s27p9.html [Consultado el 28 de marzo de 2012].

32. Moeller MR, Hartung M, Wilske J. Prävalenz von Drogen und Medikamenten bei verkehrsauffälligen Kraftfahrern. Blutalkohol 1999; 36 (1): 25-38.

33. Bratzke H, Souchon F, Kauert G. Zur Dunkelziffer drogen- bzw. medikamentenbedingter Beeinflussung bei Verkehrsdelikten und anderen Straftaten-eine Analyse von 232 "Diskrepanzfällen". Berichte Bundesanstalt für Straßenwesen 2005; 171: 149-51.

34. Decreto 404, Ministerio de Salud. Publicado en Diario Oficial el 20 de febrero de 1984. Disponible en: http:// www.leychile.cl/Navegar?idNorma $=13057 \&$ buscar $=$ dec reto $+404+1984$ [Consultado el 28 de marzo de 2012].

35. Decreto 405, Ministerio de Salud. Publicado en Diario Oficial el 20 de febrero de 1984. Disponible en: http:// www.leychile.cl/Navegar?idNorma $=13066 \&$ buscar $=\mathrm{dec}$ reto $+405+1984$ [Consultado el 28 de marzo de 2012].

36. Decreto 867, Ministerio del Interior. Publicado en Diario Oficial el 19 de febrero de 2008. Disponible en: http://www.leychile.cl/Navegar?idNorma=269323 [Consultado el 28 de marzo de 2012].

37. Decreto 170, Ministerio de Transportes y Telecomunicaciones. Publicado en Diario Oficial el 02 de enero de 1986. Disponible en: http://www.leychile.cl/ Navegar?idNorma=10426 [Consultado el 28 de marzo de 2012].

38. Blencowe T, Pehrsson A, Lillsunde P. Analytical evaluation of oral fluid screening devices and preceding selection procedures Disponible en: http://www.rosita. org/ [Consultado el 28 de marzo de 2012].

39. Resolución exenta 8833, Dirección Nacional Servicio Médico Legal. Publicada en Diario Oficial el 30 de septiembre de 2010. Disponible en: http://www.leychile.cl/ Navegar?idNorma=1017485 [Consultado el 28 de marzo de 2012].

40. Villanueva E. Estudio Toxicológico y médico legal del alcohol etílico. En Gisbert Calabuig JA., Editor. Medicina Legal y Toxicológica. Barcelona, España: Editorial Masson; 2004. p. 878-95.

41. Devleeschouwer N, Libeer JC, Martens FK, Neels H, Van Damme M, Verstraete A, et al. Blood alcohol testing: comparison of the performance obtained with the different methods used in the Belgian external quality assessment schemes. Clin Chem Lab Med 2004; 42 (1): 57-61.

42. Diagnosis. En Fletcher RH, Fletcher SW, Wagner EH, Editores. Clinical Epidemiology. The essentials. Baltimore, Maryland, Estados Unidos: Editorial Lippincott Williams \& Wilkins; 2005. p. 43-74.

43. Streiner D. A checklist for evaluating the usefulness of rating scales. Can J Psychiatry 1993; 38 (2): 140-8.

44. Jaeschke R, Guyatt GH, Sackett DL. User's guide to the medical literature. III. How to use an article about a diagnostic test. A. Are the results of the study valid? JAMA 1994; 271 (5): 389-91.

45. Designing Studies of Medical test. En Hulley SB, Cummings SR, Browner WS, Grady DG, Newman TB, Editores. Designing Clinical Research. Philadelphia, Estados Unidos: Editorial Lippincott Williams \& Wilkins; 2007. p. 183-206.

46. Oxford Centre for Evidence-based Medicine. Levels of Evidence and Grades of Recommendation (March 2009). Disponible en: http://www.cebm.net/?o=1025 [Consultado el 28 de marzo de 2012].

47. Screenning. En Hennekens CH, Buring JE, Editores. Epidemiology in Medicine. Philadelphia, Estados Unidos: Editorial Lippincott Williams \& Wilkins; 1987. p. 32745.

48. Ley 20.074. Publicada en Diario Oficial el 14 de noviembre de 2005. Disponible en: http://www.leychile.cl/ Navegar?idNorma $=243832$ [Consultado el 28 de marzo de 2012].

49. Wehner HD. Fahrtüchtigkeit. Alkohol. En Madea B, Editor. Praxis Rechtsmedizin. Befunderhebung. Rekonstruktion. Begutachtung. Berlín, Heidelberg, Alemania: Editorial Springer; 2006. p. 421-46. 